\title{
Comparison of the far-field aerodynamic wake development for three DrivAer Model configurations using a cost-effective RANS simulation
}

\author{
Renan F. Soares, Kevin. P. Garry, Jennifer Holt \\ Applied Aerodynamics Group, Cranfield University, UK
}

\begin{abstract}
The flow field and body aerodynamic loads on the DrivAer reference model have been extensively investigated since its introduction in 2012. However, there is a relative lack of information relating to the models wake development resulting from the different rear-body configurations, particularly in the far-field.
\end{abstract}

Given current interest in the aerodynamic interaction between two or more vehicles, the results from a preliminary CFD study are presented to address the development of the wake from the Fastback, Notchback, and Estateback DrivAer configurations. The primary focus is on the differences in the far-field wake and simulations are assessed in the range up to three vehicle lengths downstream, at Reynolds and Mach numbers of $5.2 \times 10^{6}$ and 0.13 , respectively.

Wake development is modelled using the results from a ReynoldsAveraged Navier-Stokes (RANS) simulation within a computational mesh having nominally $1.0 \times 10^{7}$ cells. This approach was chosen to reflect a simple, cost-effective solution, using an industry-standard CFD solver. Each vehicle configuration has a smooth underbody, with exterior rear-view mirrors. The computational modelling includes a ground simulation set, and all simulations are for zero freestream yaw angle. A mesh sensitivity study was undertaken and the simulation validated against published experimental data for the body pressure distribution and aerodynamic drag.

Critical assessment of the results highlights the benefits of focussed mesh refinement and specific numerical strategies for optimum performance of the CFD solver. Comparison of the far-field aerodynamic wake for the three model configurations exhibits significant differences in both extent and structure within the wake region up to three vehicle lengths downstream of the base. Total pressure loss coefficient is used as the primary aerodynamic parameter for analysis. The study is an element of a larger programme related to vehicle wake simulation and strategies are identified for possible wake modelling using simplified, computationally and experimentally efficient, shapes.

\section{Introduction}

In earlier years of research on automotive aerodynamics the Ahmed body [1] provided significant findings to support industrial designs and academic concepts. Beyond the well-known aerodynamic features discovered from investigations of this model and its variants, the importance of reference models to both academic and industrial researchers was highlighted, in order to develop long-term, reliable technical studies.

More recently the DrivAer model [2][3] has proven to be a widely adopted configuration, which expands the limits of previous automotive reference geometries since it is aligned to the current level of technological advances and challenges of experimental and computational tools. Most of published research relating to this model has addressed experimental testing [2][4][5][6][7][8], computational simulations and numerical techniques [4][8][9][10][11][12][13][14], and flow manipulation [15][16][17][18]. Although some publications specifically address the aerodynamic wake, the data generated, for the three models variants, does not provide much information relating to wake development beyond 0.5 vehicle body length (L) downstream.

As a consequence, this paper contains computational simulations, comparison and analysis of extended wakes generated by the Fastback, Notchback, and Estateback DrivAer models. The aim is to explore the options for using relatively cost-effective RANS simulations as a tool to analyse vehicle aerodynamic interference.

\section{Methodology}

\section{Mathematical Models}

The fluid dynamic modelling adopted is based on the assumption of incompressible flow, which is acceptable given that the chosen freestream conditions correspond to a Mach number (M) of 0.13 , at which compressible effects can be negligible [19]. The ReynoldsAveraged Navier-Stokes (RANS) approach has been adopted which means that the modelling only considers time-averaged phenomena.

Therefore, incompressible conservation of mass and Navier-Stokes equations are, respectively:

$$
\begin{gathered}
\frac{\partial u_{i}}{\partial x_{i}}=0 \\
\frac{\partial u_{i}}{\partial t}+\frac{\partial}{\partial x_{j}}\left(u_{i} u_{j}\right)=-\frac{\partial P}{\partial x_{i}}+\vartheta \frac{\partial^{2} u_{i}}{\partial x_{j} \partial x_{j}}
\end{gathered}
$$

The science of turbulent flows still does not provide an analytical function between the drag force $\left(F_{D}\right.$, or $\left.D\right)$ and the geometric shape of a vehicle. It is known that aerodynamic loads are proportional to

Page 1 of 13

Published by SAE. This is the Author Accepted Manuscript issued with: Creative Commons Attribution Non-Commercial License (CC:BY:NC 4.0). The final published version (version of record) is available online at DOI:10.4271/2017-01-1514. Please refer to any applicable publisher terms of use. 
the vehicle size, the density of the ambient air and to the square of the relative air speed. This freestream velocity $\left(U_{\infty}\right)$ can be defined as vectorial difference between wind $\left(U_{w}\right)$ and vehicle velocities $\left(U_{V}\right)$. The vehicle size is conventionally expressed in terms of the projected frontal area $\left(A_{\text {ref }}\right)$. Hence, a formal equation of drag is:

$$
F_{D} \propto \rho_{\infty} A_{r e f}\left(U_{w}-U_{V}\right)^{2}
$$

The conventional non-dimensional drag (drag coefficient, $C_{D}$ ) is adopted in this work:

$$
C_{D}=\frac{F_{D}}{\frac{1}{2} \rho_{\infty} A_{\text {ref }} U_{\infty}^{2}}
$$

Pressure coefficient $\left(C_{P}\right)$ is the pressure difference at a point relative to the free stream static pressure, normalised by the dynamic pressure of the freestream flow, resulting in:

$$
C_{P}=\frac{\left(P-P_{\infty}\right)}{\frac{1}{2} \rho_{\infty} U_{\infty}^{2}}
$$

According to fundamentals of aerodynamics, the net surface integral of total pressure in a control volume domain is proportional to the drag generated by an immersed body [21]. Assuming that the contribution of potential contribution due to gravitational force is taking into account by the static pressure, total pressure is defined by:

$$
P_{T}=P_{S}+P_{D}=P+\frac{1}{2} \rho u^{2}
$$

Thus, a total pressure loss coefficient $\left(C_{P_{T L}}\right)$ can be derived, expressed as:

$$
C_{P_{T L}}=1-C_{P_{T}}=1-\left(\frac{P}{\frac{1}{2} \rho U_{\infty}^{2}}+\left(\frac{u}{U_{\infty}}\right)^{2}\right)
$$

\section{Car Geometry}

The DrivAer model was adopted in this study and used without modifications since the reference CAD components are available on the TUM website [3]. For the purpose of this study, three rear-end variations of the model are compared: fastback, notchback, and estateback. Apart from the rear-end geometry, all car assemblies share the same smooth underbody, side mirrors, wheels with realistic rims and tread-less tyres.

In addition to the body being tested, experimental studies require a model mounting system. In the majority of the DrivAer body test cases, an aerodynamically profile overhead support strut is used to connect the model [2][5][8]. This component is not always described precisely in published works. In the simulations presented here (which will be used for validation purposes, particularly relating to the pressure coefficient distribution on the upper surface) a generic overhead strut was adopted. The strut has a NACA0025 profile, $c=$

Page 2 of 13
$0.375 \mathrm{~m}$, where the leading edge is positioned at $x=1.393 \mathrm{~m}$ on the original full-scale DrivAer coordinate system. Table 1 summaries the vehicle components used.

Table 1. Vehicle geometry.

\begin{tabular}{lcc}
\hline Component & Description & Symbol \\
\hline $\begin{array}{l}\text { Car } \\
\text { simplification } \\
\text { Rear end }\end{array}$ & Symmetrical closed half-car model & half- \\
Mirrors & Fastback, Notchback, Estateback & F, N, E \\
Underbody & Standard mirror & wM \\
Wheels & $\begin{array}{c}\text { Smooth underbody } \\
\text { Smooth version: }\end{array}$ & S \\
Strut & $\begin{array}{c}\text { generic rims and with no tire treads } \\
\text { with Top Strut (NACA 0025), } \\
\text { with no wheel struts }\end{array}$ & wW \\
\hline
\end{tabular}

Geometric dimensions have been parametrized as a function of the car scale $(C S)$. Due to most of the available experimental data being related to a $40 \%$ scale-model, the same car scale is adopted in this study [2][5][8]. Table 2 presents the DrivAer car dimensions as a function of the car scale, as well as the respective values assumed in this paper.

Table 2. Proposed parametric dimensions for the DrivAer car model.

\begin{tabular}{lccrc}
\hline Parameter & Symbol & Definition & Value & \\
\hline Car scale & $C S$ & - & $40 \%$ & \\
Reference length & $L$ & 4.6126 CS & 1.8450 & $m$ \\
Front end x-position & $x / L=0$ & $-0.8075 C S$ & {$[-0.323,0,0]$} & $m$ \\
Front wheels axle position & $x_{F}$ & 0 & {$[0,0,0]$} & $m$ \\
Rear wheels axle position & $x_{R}$ & $2.7861 C S$ & {$[1.1145,0,0]$} & $m$ \\
Reference height & $H$ & 1.4182 CS & 0.5673 & $m$ \\
Reference width; & $W$ & $1.7529 C S$ & 0.7012 & $m$ \\
wheeltrack & $W R$ & $0.3180 C S$ & 0.1272 & $m$ \\
Wheel radius & $W B$ & 2.7862 CS & 1.1145 & $m$ \\
Wheelbase & $A_{r e f}$ & $\begin{array}{r}2.1605 C S^{2} \\
\text { (full-car, F) }\end{array}$ & $\begin{array}{r}0.1728 \\
\text { (half-car) }\end{array}$ & $m^{2}$ \\
Reference area & & & & \\
\hline & & &
\end{tabular}

\section{Fluid Domain}

In all cases, the numerical domain consists of the symmetrical half of the fluid domain on the positive $y$-coordinate side, considering that the +ve $\mathrm{x}$-coordinate is in the downstream direction, and the +ve $\mathrm{z}$ coordinate is in the lift direction. This coordinate system shares the same orientation as that presented in Hucho [20], Katz [22], and Barnard [23]. The global coordinate system origin is the same as the DrivAer CAD geometries - the centre point of the front wheel axle.

Air is the working fluid with properties: temperature $(\mathrm{T})=298 \mathrm{~K}$, density $(\rho)=1.18415 \mathrm{~kg} / \mathrm{m}^{3}$, and the dynamic viscosity $(\mu)=$ $1.85508 \times 10^{-5} \mathrm{Ns} / \mathrm{m}^{2}$. Freestream conditions correspond to a $R e=$ $5.2 \times 10^{6}$, which is equivalent to $44.2 \mathrm{~m} / \mathrm{s}$ and Mach number of 0.13 . 
The computational domain over the half-body is a slab of width equal to $5.7 \mathrm{~W}$ from the symmetry plane, height of $7 \mathrm{H}$ from the ground, and a total length of $13 L$ [9][11][15]. The area blockage ratio in these cases is $\approx 1 \%$. Vehicle positioning is done by placing the flow inlet $2 L$ upstream the coordinate origin. Positioning of the ground required a decision to be made relating to the wheel-surface contact. In order to replicate the physical fluid phenomena around the wheel-surface (e.g. realistic pressure distribution and corresponding vortex flowfield), it is necessary to replicate a realistic contact patch [10][12]. The approaches considered in this case are as follows: (a) created and fix the lowest plane tangential to the tyres as the ground plane, then slightly cross it with the wheels geometry, or (b) maintain the original positioning of body and wheels, and cross the wheels surfaces with lowest tangent plane (i.e. ground) moving it upwards. Even though both approaches would provide the same tyre patch, the first approach would change the position of the wheel inside the wheelhouse, and it is known to be a region of significant drag generation. As a result the second solution (b) has been adopted since the positioning between body and wheelhouse is sustained (see Figure 1), whereas a small reduction in the body ground clearance ( $<0.01 \mathrm{~m}$ in full-scale) may result in a small increase in ground effects (i.e. increase of local flow velocity coupled to a pressure reduction). This is considered a necessary consequence of sustaining the geometry similarity inside wheelhouses and the related fluid dynamics from such regions. For all simulations, the ground plane is set $0.219 \mathrm{H}$ below the front wheel axle $(0.31 \mathrm{~m}$ in full-scale).

Table 3 summarises the normalised parameters regarding the fluid domain for both dimensional and scalar properties.
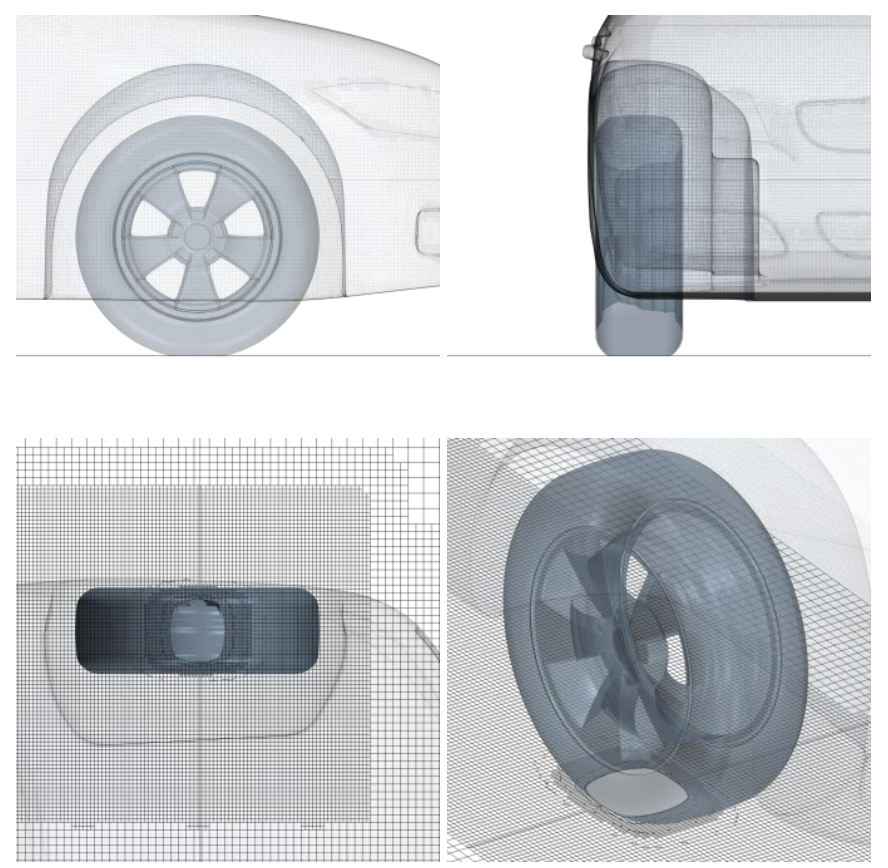

Figure 1. Frontal tire contact patch. Car and ground surfaces illustrate the meshing distribution resulting from the meshing process.

Page 3 of 13
Table 3. List of normalised parameters for the DrivAer body.

\begin{tabular}{lcc}
\hline Parameter & Symbol & Definition \\
\hline Normalised x-coordinate & $x / L$ & $(x-0.8075 C S) / L$ \\
Normalised y-coordinate & $y / W$ & $y / W$ \\
Normalised z-coordinate & $z / H$ & $z / H$ \\
Normalised velocity & $u / U_{\infty}$ & $u / U_{\infty}$ \\
Drag coefficient & $C_{D}$ & $F_{D} /\left(\rho_{\infty} A_{r e f} U_{\infty}{ }^{2} / 2\right)$ \\
Lift coefficient & $C_{L}$ & $F_{L} /\left(\rho_{\infty} A_{r e f} U_{\infty}{ }^{2} / 2\right)$ \\
Pressure coefficient & $C_{P}$ & $P /\left(\rho_{\infty} U_{\infty}{ }^{2} / 2\right)$ \\
Total pressure coefficient & $C_{P_{T}}$ & $\frac{P}{\rho_{\infty} U_{\infty}{ }^{2} / 2}+\left(\frac{u}{U_{\infty}}\right)^{2}$ \\
Total pressure loss coefficient & $C_{P_{T L}}$ & $\left(1-C_{P_{T}}\right)$ \\
\hline
\end{tabular}

\section{Numerical Tool}

All numerical simulations presented in this paper are carried out using Star-CCM+ version 11.02, double-precision. The CFD setup employed is mostly as a result of experience gained from previous studies [9][10][11][15][18]. Such a numerical simulation had been optimised aiming at a simple, higher cost-benefit of computational resources for automotive aerodynamics, which is based on RANS modelling in steady-state condition and constant density. The flow solver is segregated in order to reduce numerical instabilities. Turbulence properties are modelled by a robust version turbulence model from the $k-\varepsilon$ group: Realisable $k-\varepsilon$ two-layer. All numeric gradients are computed in $2^{\text {nd }}$-order precision, Hybrid Gauss- $L S Q$. For the purpose of reducing computational requirements linked to higher meshing resolution in boundary layer modelling, All y+ Wall Treatment is adopted to deal with prism layers described later. Table 4 summarises the main CFD setup.

Table 4. CFD setup summary.

\begin{tabular}{lc}
\hline Boundary & Condition \\
\hline Domain simplification & half-symmetrical three-dimensional \\
Time regime & Steady-state \\
Equation of State & Constant density \\
Reynolds Number & $R e=5.2 \times 10^{6}$ \\
Fluid & Air $\left(\rho=1.18415 \mathrm{~kg} / \mathrm{m}^{3} ; \mu=1.85508 \times 10^{-5} \mathrm{Ns}^{\circ} \mathrm{m}^{2}\right)$ \\
Flow Solver & Segregated \\
Flow regime & Turbulent \\
Turbulence model & Realisable $k$ - $\varepsilon$ two-layer \\
Gradient order & $2^{\text {nd }}$-order, Hybrid Gauss-LSQ \\
\hline
\end{tabular}

\section{Boundary Conditions}

The inlet surface is set as a velocity-constant inlet, while the outlet surface is set as a pressure-constant outlet. The surface in the symmetry plane, as the farfield boundary (i.e. top and side surfaces), are set to mirror properties (e.g. symmetry condition). Ground simulation is considered in the modelling, so the ground has a relative velocity to the car that matches the freestream velocity from the inlet. 
Wheel modelling for ground simulations remains a challenge for CFD techniques. However, it has been shown that imposing a tangent velocity on the wheel surface can be an acceptable approach since it emulates similar effects from wheel motion in a time-averaged matter. Similarly, it also avoids the need for time-dependent remeshing, (e.g. unsteady-state simulation) for averaged-results. Details and samples are available at [9][10][11]. An equivalent approach is adopted in this paper, two boundaries are created to represent each pair of wheels: front and rear wheels. A tangential velocity field on the each wheel surface is imposed by applying a local rotational rate $\left(\omega_{w}\right)$ on each pair of wheels, calculated as the ratio of relative ground velocity $\left(U_{G S}\right)$ and wheel radius $(W R)$. Parameters of each boundary are presented and summarised in Table 4.

Table 4. Boundary conditions.

\begin{tabular}{ccc}
\hline Boundary & Condition & Parameters \\
\hline Car & wall & no-slip \\
Inlet & velocity-inlet & $U_{\infty}=44.2 \mathrm{~m} / \mathrm{s} ; \quad I=0.5 \% ; \mu_{t} / \mu=2$ \\
Outlet & pressure-outlet & $P_{\infty}=0 \mathrm{~Pa} ; \quad P_{a b s}=101325 \mathrm{~Pa}$ \\
Sym & Symmetry & \\
Farfield & symmetry & \\
Ground & wall & no-slip, ground movement at $U_{\mathrm{GS}}=44.2 \mathrm{~m} / \mathrm{s}$ \\
Wheels & wall & no-slip, local rotation rate at $\omega_{\mathrm{w}}=347.5 \mathrm{rad} / \mathrm{s}$
\end{tabular}

\section{Meshing process}

Discretization of the fluid domain has been done by splitting it predominantly into trimmed cells type. A moderately aligned main flow and lower RAM memory use (i.e. one of the key computational resources) are two of the reasons for this choice. A proper simulation of relevant fluid dynamic characteristics, such as recirculation flow and fully turbulent zones, would require a small increase in a number of cells when compared to polyhedral cell type.

Prism layers are used on wall surfaces that are expected to develop boundary layers. As wall functions are available, all first prism layers are adjusted to fit $y^{+}$more than 30 (i.e. minimum threshold of the log-law section into the inner region of boundary layers). For these cases, the first prism layer thickness is $0.5 \mathrm{~mm}$, and the total prism region of four layers has a thickness of $6.0 \mathrm{~mm}$. Ground surface is an exception since all simulations consider ground velocity as being the same as the freestream, thus any boundary layer far from the vehicle would be minimal. Only one prism layer is set on the ground boundary with a thickness equal to $2.4 \mathrm{~mm}$. This is not linked to capturing details of a marginal relative boundary layer, but aims at vertical refinement on the ground wherever the meshing become considerably coarse (i.e. better properties interpolation between ground and freestream in both intel and outlet contact edges to the ground).

According to Soares [9][10][11], it has been found that $10 \mathrm{~mm}$ on a full-scale DrivAer model must be the highest threshold when regarding the meshing cell size on car surface meshing for RANS simulations. Likewise, the reference meshing size parameter $(h \times C S)$ is proposed and adopted as the global parametric meshing dimension, where $h$ denotes the base meshing size defined as the edge size, in

Page 4 of 13
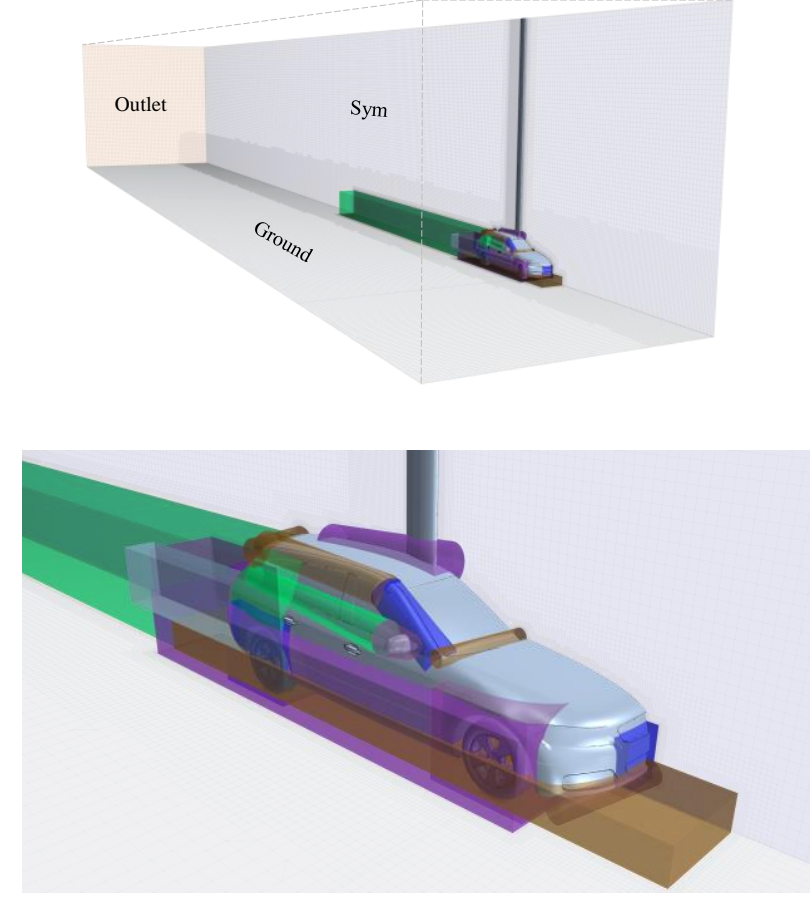

Figure 2. Numerical domain (upper) and main regions of refinement control (lower) used for meshing optimisation purpose.

$\mathrm{mm}$, of a reference cubic cell, and CS is the car scale. Meshing efficiency goes beyond the prism layer and surface resolution optimisation. Higher meshing resolutions have been set as required, though minimising as much as possible any over-refinement of nonrelevant zones. In other words, refinements have been applied only as required, such as wheelhouses, mirrors, A-pillar, hood end, and trunk edge, to name a few shown in Figure 2.

In view of the primary motivation for this study, the main wakes from the each car model received a proper refinement in the meshing process, and have been split into 4 regions: base wake core, rear bumper vortex core, wheels wake, and extended wake. The first three have been set under the same resolution of $4 \mathrm{~mm}(1.0 \mathrm{~h} \times C S)$ into their respective zones. This is not undertaken on the basis of previous research, but has been added in order to provide a reasonable resolution of the extended wake properties, and consequently improved wake assessment by sustaining a resolution of $8 \mathrm{~mm}$ $(2.0 h \times C S)$ for at least three car lengths. In addition to these four regions, frontal car area blockage of $25 \%$ and resolution of $8 \mathrm{~mm}$ $(8.0 h \times C S)$.

In summary, the computational mesh of each case has nominally $1.0 \times 10^{7}$ cells. Due to the over-refinement in the wake core up to three car lengths, it is twice the number recommended by Soares [9][11]. However, in the case of a RANS simulation, the meshing strategy required only a half of the number used by Heft et al. [4] and 1/8 of Ashton et al.'s CFD cases [13][14].

\section{Validation process}

Validation process had evaluated the capability of the numerical tool in predicting the flow field surrounding an automotive geometry. A mesh sensitivity study was carried out in order to ensure the meshing requirements described in the CFD setup proposed by Soares [9]. 
Figure 3 shows the influence of meshing resolution on the drag coefficient of the DrivAer Fastback, in ground simulation. Numerical results distinguish the increment of drag coefficient from different car parts, such as wheels, mirrors, top strut, and body (e.g. car base + rear end). Two published experimental databases, from the Audi and TUM wind tunnels, are compared to the numerical outcomes from the car assembly [8]. As expected, drag coefficients tend to asymptotic magnitudes as the mesh refinement level reduces. The results indicate that the meshing resolution suitable for aerodynamic loading prediction should be no more than $h=15$. Pressure-based drag components (e.g. body) are less sensitive the mesh resolution, while the viscous drag component (e.g. top strut aerofoil) is highly sensitive to spatial discretization.

Beyond a sensitivity analysis of aerodynamic loading, an additional assessment of the wake itself was performed for the purpose of appropriate wake comparison. Figure 4 shows the influence of the meshing resolution on measurements of total pressure coefficient taken from six numerical probes positioned in the wake. Each probe group evaluated three position planes downstream the wake: $1.50 \mathrm{~L}$, 2.00L, and 4.00L; covering two planes: one in the plane of symmetry (i.e. $y / W=0$ ), and another $y / W=0.25$. All probes are at the same height as the front wheel axle (i.e. $z / H=0$ ).

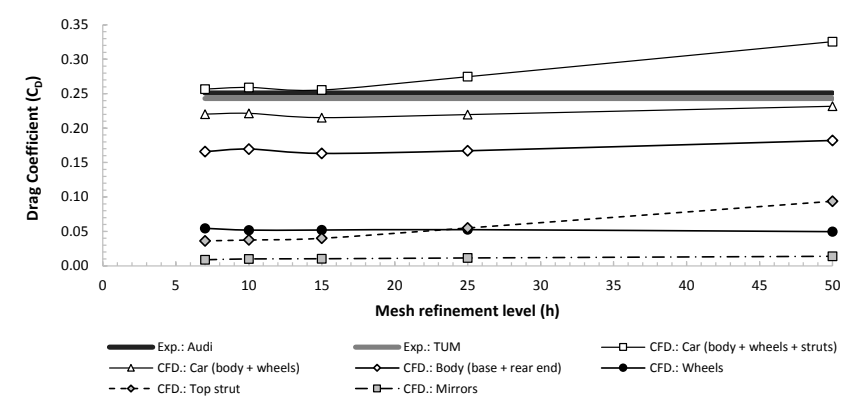

Figure 3. Meshing sensitivity of the car surface: distribution of drag coefficient $\left(C_{D}\right)$ on a Fastback model (FSwMwW, GS on, wTS).
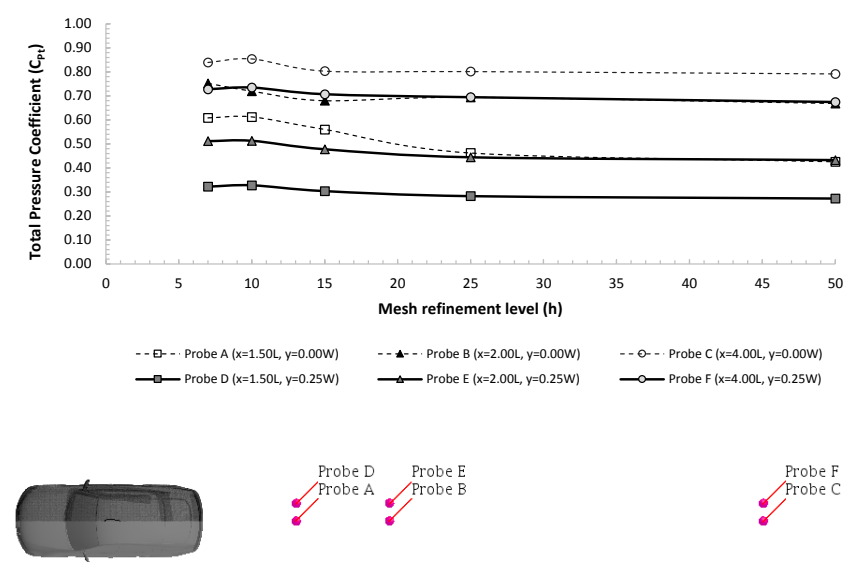

Figure 4. Meshing sensitivity of the wake: total pressure coefficient $\left(C_{P_{T}}\right)$ measured by six numerical probes, downstream a Fastback model (FSwMwW, GS on, wTS).

Page 5 of 13
Interestingly, the wake characteristics show a different tendency to that of the aerodynamic loading. In the earlier refinements from the coarser mesh, a considerable increment in the drag is apparent in contrast to the minimal wake variation (see Figure 4). Finer meshes had indicated higher deviation of the total pressure, mainly from $h=$ 15 to 10 . A possible explanation for the behaviour is that better property gradients are revealed as much as a mesh is refined, since discretization of continuum properties often hides numerical picks on the interpolation process (e.g. meshing discretization).

Therefore, aerodynamic load assessment may require a maximum meshing refinement level of 15 , whilst wake properties seem to need no more than $h=10$. This highlights the relevance of meshing sensitivity analysis on the wake whenever wake interaction might be concerned. Additionally, the results confirm that a mesh refinement level of 10 is fine enough to reasonably assess the aerodynamics of automotive shapes, as proposed in earlier studies [9][11].

Based on the validation process, the drag coefficient breakdown for the fastback, notchback, and estateback configurations are presented in Figure 5.

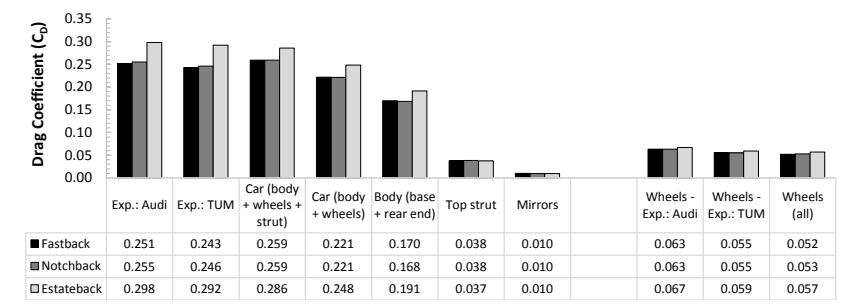

Figure 5. Geometry sensitivity: comparison of drag coefficient $\left(C_{D}\right)$ distribution among Fastback, Notchback, Estateback car models simulated with a top strut (_SwMwW, GS on, wTS).

\section{Analysis of the Aerodynamic Wakes}

The subsequent sub-sections aim to illustrate wake characteristics via both velocity and total pressure loss. All assessments were based on extracting properties from equidistant, perpendicular planes to the freestream. Wakes were analysed using data from 13 planes over a range of three car lengths, from the first plane at the rear of the vehicle $(x / L=1.0)$ followed by other by constant gap of $0.25 L$ (i.e. wake evaluated from $1.00 \mathrm{~L}$ up to $4.00 \mathrm{~L}$ ). Note that the x-position has been normalised by the vehicle length, and its origin lies at the front of the vehicle.

\section{Wake characteristics based on the velocity vector field}

Vortex cores have been extracted from their respective car wake. The criterion adopted to identify each vortex core path is purely kinematic since it has been defined here as the rotational centre point exposed by velocity vectors (i.e. $u_{y}$ and $u_{z}$ components) from each cross section, with regards to the main coherent recirculation.

Figure 6 presents a comparison of the dominant vortex core from the fastback, notchback, and estateback models under identical conditions. Due to its three-dimensional displacement, the vortex 


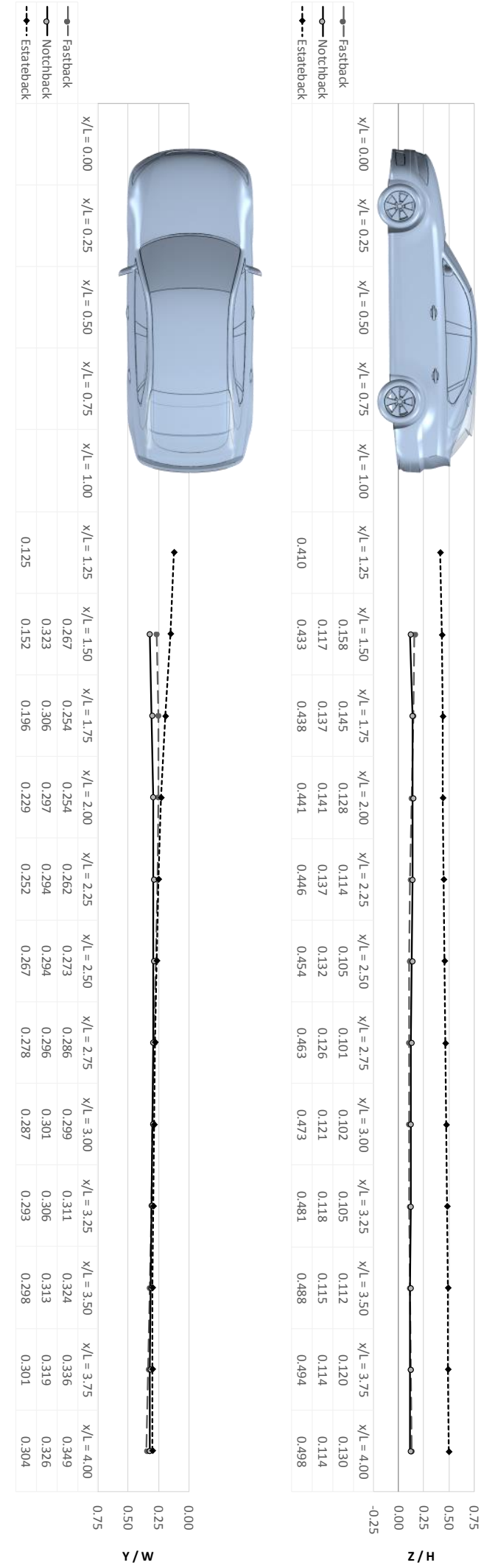

Figure 6. Comparison of the vortex core (based on velocity vector field) in the wake generated by Fastback, Notchback, Estateback car models (_SwMwW, GS on, wTS). Dimensions are normalised by the car length $(L)$ and height $(H)$ in the respective directions. core path is shown in projected planes (xz and yz) using normalised dimensions.

As expected from the loading coefficients, the fastback and notchback configurations generate a similar main vortex path in their wakes, while the estateback shows different trend.

The top view (xy plane projection, Figure 6, at left-hand side) shows that the main vortex can be identified from the plane at $\mathrm{x}=1.50 \mathrm{~L}$ for the fastback and notchback configurations, compared to a distance of $0.25 \mathrm{~L}$ for the estateback configuration. The fastback vortex path firstly appears in the plane $\mathrm{x}=1.50 \mathrm{~L}$ at $\mathrm{y}=0.267 \mathrm{~W}$ from the symmetry plane, decreases by approximately $5 \%$ at $2.00 \mathrm{~L}$, and expands continuously downstream. For the notchback wake, it follows similar trend from fastback, although it starts further outboard $(0.323 \mathrm{~W})$ and generates minor contraction and expansion variations during its progression downstream. In contrast, the estateback generates a vortex path from $0.125 \mathrm{~W}$ at $1.25 \mathrm{~L}$ and expands rapidly up to $0.267 \mathrm{~W}$ at $2.50 \mathrm{~L}$, and then more slowly up to the plane at $\mathrm{x}=4.00 \mathrm{~L}$.

The side view (xz plane projection, Figure 6, at right-hand side) clearly illustrates the different wake development for the estateback configuration compared to the other two shapes. Note that the zcoordinate origin is the front wheel axle. The fastback geometry generates a vortex nearer to the rear bumper height $(0.158 \mathrm{H}$ at $1.50 \mathrm{~L}$ ), which fluctuates between $\mathrm{z}=0.10 \mathrm{H}$ and $0.13 \mathrm{H}$ as it is carried downstream. The notchback configuration shows a similar vortex height, however with an opposite initial trend: initial upwash cycle from $\mathrm{z}=0.117 \mathrm{H}$ at $1.50 \mathrm{~L}$ until $\mathrm{z}=0.141 \mathrm{H}$ at $2.00 \mathrm{~L}$, and finally deflected downwards progressively to $\mathrm{z}=0.114 \mathrm{H}$ at $\mathrm{x}=4.00 \mathrm{~L}$. In sharp constrast, the main coeherent vortex structure is advected almost twice as high from the ground compared to the other configurations, beginning at $\mathrm{z}=0.41 \mathrm{H}$ at $\mathrm{x}=1.25 \mathrm{~L}$ and expanding gradually to $\mathrm{z}=0.498 \mathrm{H}$ by the last plane evaluated.

In summary, two wakes groups can be identified in terms of the vortex path: (i) fastback and notchback, and (ii) estateback. In a top view, all vortex cores extend laterally to $\mathrm{y} \approx W / 3$ by the plane at $\mathrm{x}=$ $4.00 \mathrm{~L}$, while the estateback vortex path is nearly twice as high as those from the fastback and notchback configurations throughout the wake.

\section{Wake characteristics based on total pressure loss coefficient}

The total pressure loss coefficient, Eq. (7), is used in order to expand the present wake comparison for the DrivAer variant models.

Magnitude and physical location, relative to the vehicle, are the main characteristics discussed in this sub-section.

The main wake is generated by flow separation behind the body, as a result analysis of the first plane at $x / L=1.00$ provides insight into the wake generation. All configurations indicate that the most significant reduction in total pressure (i.e. $C_{P_{T L}}>1.0$ ) is mainly restricted to a projected area corresponding to the base rear end and rear wheels, although the latter is translated towards to the symmetry plane by a distance of approximately one wheel width. In addition to this primary region there is evidence of the wake from the side mirror in all cases (i.e. $C_{P_{T L}} \approx 0.2$ ). In addition a wake from the top strut is 
captured (i.e. $C_{P_{T L}} \approx 0.1$ ). There is a thin layer on the ground where $C_{P_{T L}}<0.05$, which may be linked to both numerical residuals and minimal boundary layer developed by the accelerated flow surrounding each car model. Apart from abovementioned trends, it is possible to classify the three wake genesis patterns into two groups: (i) fastback and notchback, and (ii) estateback.

Both the fastback and notchback geometries share similar characteristics, and as seen in Figure 7 and Figure 10 the dominant region of the respective wake is generated from the rear end and wheels region. The only main differences apparent on the notchback case, at $x / L=1.00$, are slightly more intense trace from the rear bumper corner, and more segregated side mirror wake trace from the main wake. In contrast the estateback model generates a wider, more intensive area of total pressure loss. Figure 7 shows that the region of higher loss is nominally $10 \%$ greater that both the fastback and notchback. Beyond the rear base end and wheels, it includes the rear window and extends almost to the roof-end spoiler and D-pillar projections. The wake from the side mirror is also present in similar intensity to the other models, however, it is wider and more separated from the main region, and positioned marginally lower. The evidence that the shear layer from the flow separation behind the vehicles bounded most of the region regarding total pressure loss.

Beyond the differences in the distribution of total pressure loss at the wake onset, planes downstream the wake reveal consequential differences on the wake evolution. Both the magnitude and shape of the total pressure loss distribution along the wakes suggest that total pressure loss is reduced exponentially downstream. All planes at $x / L$ $=1.25$, exhibit significant differences to those at $x / L=1.00$ first cross section. Variations of pressure loss magnitude appear more significant than the distribution location itself when assessing planes downstream of $x / L=1.50$. Nevertheless, there are significant differences among the wakes from each DrivAer variant.

The main wake of the most aerodynamic shape, the fastback model, is rapidly dispersed from the initial near-rectangular form to a dualcircular trace up to $x / L=2.00$ (i.e. one car length). Such circular traces are linked to counter-rotating vortices that dominate the wake downstream. The reduction of total pressure decays rapidly during the first quarter car-length distance into the wake, where the most intense region $\left(C_{P_{T L}}>0.8\right)$ is now a wheel projection towards the car centre. This occurs as the region related to the rear base, shown on $x / L=1.00$ plane, contracts towards its centre, while the inner part of the wheel projection wake near to ground is constantly convected. The losses completely reach a projected zone below the underfloor at $x / L=1.75$. The outer portion of the rear wheels and the side mirror sustain their main characteristics and location during this process along the first car-length dispersion. The side mirror wake is only evident for one vehicle length and dissipates completely between $x / L$ $=2.00$ and 3.00 .

The notchback geometry generates a similar wake development to that of the fastback, although some differences are apparent. The trace related to the counter-rotating vortex pair in the notchback case suggests they are closer to each other compared to the fastback model. Another difference is the side mirror wake from notchback car which is clearly evident for the first car-length distance, initiates a merging process with the dominant region along the second carlength distance, and still shows a residual trace when reaching a distance of three car lengths (i.e. $x / L=4.00$ ).
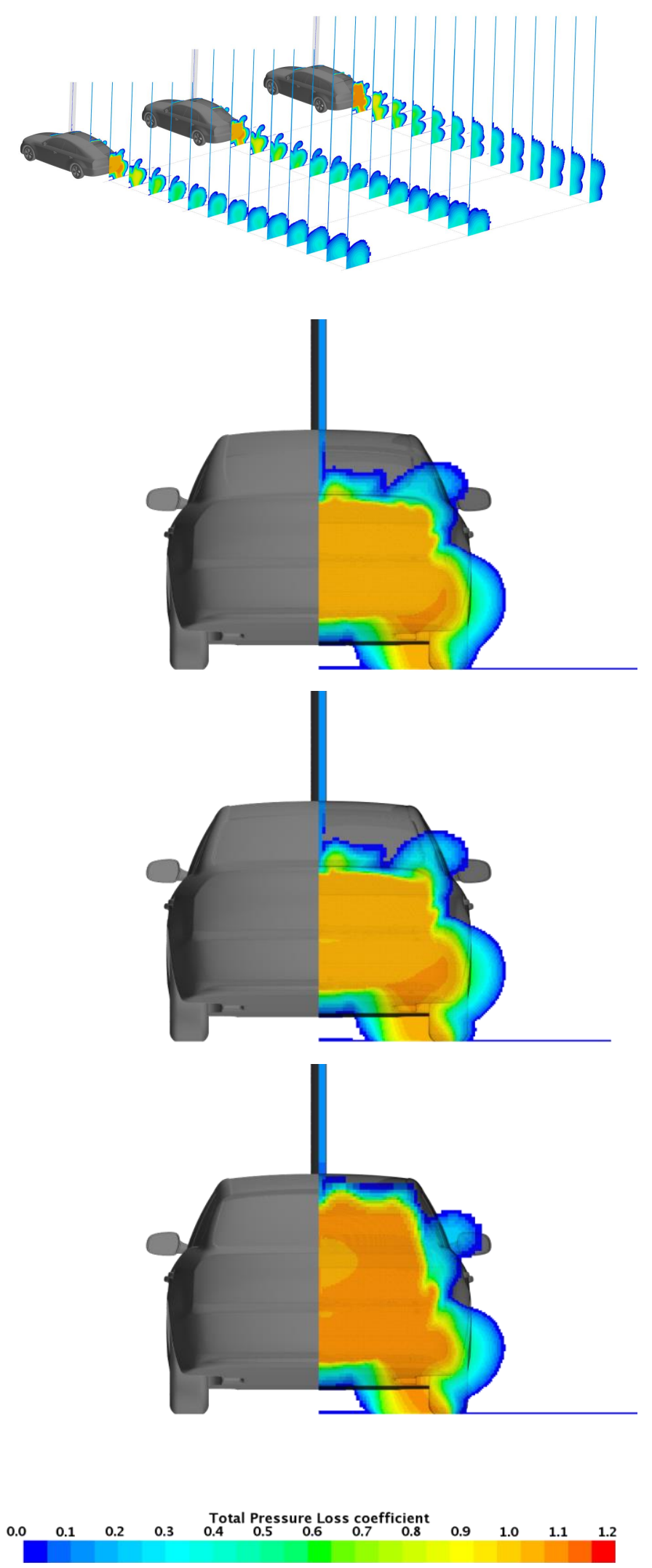

Figure 7. Total pressure loss coefficient $\left(C_{P_{T L}}\right)$ displayed just behind the fastback (upper), notchback (middle), and estateback (lower) car models, in a yz plane at $x / L=1.00$. 
Instead of a wake dominated by a low-lying, counter-rotating vortex pair, the estateback provides a considerably different wake evolution by the end of analysed wake range. Initially, the wake disperses the total pressure loss in a similar intensity to the others geometries. By the second plane (i.e. $x / L=1.25$ ), however, not only the wheel region is the new core of the pressure loss, but an additional area of higher loss is presented by the upper centre of the projected rear car. Two circular zones are now well-shaped afterwards the first car length into the wake (i.e. $x / L=2.00$ ). The lower circular trace successively decays its intensity and moves inwards. Simultaneously the upper circular trace expands predominantly upwards and by the same intensity reduction rate. By crossing the wake characteristics from Fig. 4 and 5, it becomes evident that the upper circular trace is a coherent vortex generated at the aforementioned additional region of energy loss from $x / L=1.25$.

While the wake traces generated by side mirrors are evident in the fastback and notchback wakes for at least $1.0 \mathrm{~L}$ and $1.5 \mathrm{~L}$, respectively, it is not the same for the estateback. This model generates a total pressure loss wake from the side mirror that remains relatively independent for only $0.5 \mathrm{~L}$ behind the car, and the mixing process to the main wake is complete by $2.25 \mathrm{~L}$ (i.e. $x / L=3.25$ ).

\section{Summary}

The flow field and body aerodynamic loads on the DrivAer reference model have been extensively investigated since its introduction in 2012. However, there is a relative lack of information relating to the models wake development resulting from the different rear-body configurations, particularly in the far-field.

Given current interest in the aerodynamic interaction between two or more vehicles, the results from a preliminary CFD study are presented to address the development of the wake from the Fastback, Notchback, and Estateback DrivAer configurations. The primary focus is on the differences in the far-field wake and simulations are assessed in the range up to three vehicle lengths downstream, at a Reynolds and Mach numbers of $5.2 \times 10^{6}$ and 0.13 , respectively.

Analysis of the aerodynamic wake was undertaken in terms of the wake velocity field which highlighted the presence of coherent vortices in the wake of each model configuration. Two wakes groups can be defined using the vortex path: (i) fastback and notchback, and (ii) estateback. All vortex cores reach $\mathrm{x}=4.00 \mathrm{~L}$ at approximately $\mathrm{y}=$ $W / 3$, while the estateback vortex path is nearly twice as high above the ground as those from the fastback and notchback configurations throughout the wake.

The total pressure loss coefficient becomes a useful approach for the characterization of the wakes. The highest region of pressure loss is just behind the vehicle base, and the losses decay exponentially downstream within the wake. Fastback and notchback again show similarities, however, it is possible to classify the three wake onset patterns into two group: (i) fastback and notchback, and (ii) estateback. The first group display severe dispersion from the initial rectangular-based form to a dual circular trace up to one car length. Instead of a wake dominated by a low height, counter-rotating vortex pair, the estateback provides a considerable different wake evolution by the end of analysed wake range of three car-length. The estateback wake expands its upper portion upwards while the lower portion contract inwards.

Page 8 of 13
The use of a suitable RANS CFD simulation is seen to offer the capability of a simple, cost-effective numerical approach for the assessment of the wake development from the three variants of the DrivAer car model in the range up to three vehicle lengths downstream.

\section{Contact Information}

Renan Francisco Soares

$\mathrm{PhD}$ student in Aerospace

School of Aerospace, Transport and Manufacturing (SATM)

Building 83, Cranfield University

Cranfield, Bedfordshire, MK43 0AL, United Kingdom

Email: R.soares@cranfield.ac.uk

LinkedIn: www.linkedin.com/in/renanfranciscosoares

\section{Acknowledgements}

The authors are thankful to National Council for Scientific and Technological Development $(\mathrm{CNPq})$ for the research scholarship, as well as to CD-Adapco for providing the computational tool.

\section{References}

[1] Ahmed, S.R. "Influence of Base Slant on the Wake Structure and Drag of Road Vehicles". Trans. ASME. Journal of Fluids Engineering, 105, p.429-434, 1983, doi:10.1115/1.3241024.

[2] Heft, A., Indinger, T., Adams, N. "Introduction of a new realistic generic car model for aerodynamic investigations". SAE Technical Paper 2012-01-0168, 2012, doi:10.4271/201201-0168.

[3] DrivAer Model. Geometry. Institute of Aerodynamics and Fluid Mechanics. Technical University of Munich.

[4] Heft, A., Indinger, T., Adams, N. "Experimental and Numerical Investigation of the DrivAer Model". ASME 2012, July 8-12, 2012, Puerto Rico, USA, FEDSM2012-72272. doi:10.1115/fedsm2012-72272.

[5] Mack, S., Indinger, T., Adams, N., Blume, S., Unterlechner, P. "The Interior Design of a 40\% Scaled DrivAer Body and First Experimental Results". ASME 2012, July 8-12, 2012, Puerto Rico, USA, FEDSM2012-72371. doi:10.1115/fedsm2012$\underline{72371 .}$

[6] Strangfeld, C., Wieser, D., Schmidt, H., Woszidlo, R. et al. "Experimental Study of Baseline Flow Characteristics for the Realistic Car Model DrivAer". SAE Technical Paper 2013-011251, 2013, doi:10.4271/2013-01-1251.

[7] Wieser, D., Schmidt, H., Müller, S., Strangfeld, C. et al. "Experimental Comparison of the Aerodynamic Behavior of Fastback and Notchback DrivAer Models". SAE Int. J. Passeng. Cars - Mech. Syst. 7(2):682-691, 2014, doi:10.4271/2014-01$\underline{0613 .}$

[8] Collin, C., Mack, S., Indinger, T., Mueller, J. "A Numerical and Experimental Evaluation of Open Jet Wind Tunnel Interferences using the DrivAer Reference Model". SAE Int. J. Passeng. Cars - Mech. Syst. 9(2):657-679, 2016, doi:10.4271/2016-01-1597.

[9] Soares, R. F., Souza, F. J. "Influence of CFD Setup and Brief Analysis of Flow Over a 3D Realistic Car Model". SAE Technical Paper 2015-36-0513, 2015, doi:10.4271/2015-36$\underline{0513}$.

[10] Soares, R. F., Souza, F. J. "Influence of numerics and analysis of external flow over a three-dimensional realistic car model". THMT 2015 - 8th International Symposium on Turbulence, 
Heat and Mass Transfer, Sarajevo, 2015, doi:10.13140/RG.2.1.1068.0408.

[11] Soares, R. F. "Drag of road cars: cost-effective CFD setup, proposal of an aerodynamic concept and case studies". 2015. 197p. Master Thesis, Universidade Federal de Uberlândia, Uberlândia, 2015, doi:10.13140/RG.2.1.3591.1765, UFU.

[12] Peters, B., Uddin, M., Bain, J., Curley, A. et al. "Simulating DrivAer with Structured Finite Difference Overset Grids". SAE Technical Paper 2015-01-1536, 2015, doi:10.4271/2015-011536.

[13] Ashton, N., Revell, A. "Comparison of RANS and DES Methods for the DrivAer Automotive Body". SAE Technical Paper 2015-01-1538, 2015, doi:10.4271/2015-01-1538.

[14] Ashton, N., West, A., Lardeau. S., Revell, A. "Assessment of RANS and DES Methods for Realistic Automotive Models". Computers \& Fluids 128 (April 2016): 1-15. doi:10.1016/j.compfluid.2016.01.008.

[15] Soares, R. F., Souza, F. J. "Tailpipe Position over a Realistic 3D Road Car Model: The Effect on Drag Coefficient". SAE Technical Paper 2015-36-0522, 2015, doi:10.4271/2015-360522.

[16] Wieser, D., Lang, H., Nayeri, C., Paschereit, C. "Manipulation of the Aerodynamic Behavior of the DrivAer Model with Fluidic Oscillators". SAE Int. J. Passeng. Cars - Mech. Syst. 8(2):687-702, 2015, doi:10.4271/2015-01-1540.

[17] Wieser, D., Nayeri, C., Paschereit, C. "Experiments with Vortex Generators applied to a Notchback Car Model”. 53rd AIAA Aerospace Sciences Meeting, AIAA 2015-1236. doi:10.2514/6.2015-1236.

[18] Soares, R. F., Souza, F. J. "Proposal of an Aerodynamic Concept for Drag Reduction of Fastback Car Models". SAE Technical Paper 2015-36-0523, 2015, doi:10.4271/2015-360523.

[19] Keogh, J., Doig, G., Diasinos. S. "Flow Compressibility Effects Around an Open-Wheel Racing Car.” Aeronaut. J. 118, no. 1210 (December 2014): 1409-1431. doi: $10.1017 / \mathrm{s} 0001924000010125$.

[20] Hucho, W-H. et al. "Aerodynamics of Road Vehicles", Fourth Edition, Society of Automotive Engineers Inc, 1998. ISBN: 9780768000290.

[21] Anderson, John D. "Fundamentals of aerodynamics", $6^{\text {th }}$ edition. Boston: McGraw-Hill, 2016. ISBN: 9781259251344.

[22] Katz, J. "Race Car Aerodynamics: Designing for Speed". $2^{\text {nd }}$ edition. Cambridge: Bentley Publishers, 2006. ISBN 9780837601427.

[23] Barnard, R. H. "Road Vehicle Aerodynamic Design", 3nd edition. Cornwall: MechAero Publishing, 2009. ISBN: 9780954073473 
Definitions/Abbreviations

$A_{\text {ref }}$

c

$C_{D}$

$C_{L}$

$C_{P}$

$C_{P_{T}}$

$\boldsymbol{C}_{P_{T L}}$

CS

CFD

E

$\boldsymbol{F}$

$F_{D}, \mathrm{D}$

GS off

GS on

$\boldsymbol{h}$

$h \times C S$

H

I

$\boldsymbol{L}$

LSQ

M

$N$

RANS

Re

$\boldsymbol{P}$

$\boldsymbol{P}_{a b s}$

$\boldsymbol{P}_{D}$
Reference Frontal Area

Chord length

Drag coefficient

Lift coefficient

Pressure coefficient

Total pressure coefficient

Total pressure loss

coefficient

Car scale

Computational Fluid

Dynamics

Estateback

Fastback

Drag force

Stationary ground condition

Ground simulation

Base meshing size

Reference meshing size

Reference car height

Turbulence intensity

Reference car length

Least squared

Mach number

Notchback

Reynolds-Averaged Navier-

Stokes

Reynolds number

Pressure

Absolute pressure

Dynamic pressure
$\boldsymbol{P}_{\infty}$

$\mathbf{S}$

$T$

$u$

$u_{y}$

$\boldsymbol{u}_{\mathrm{z}}$

$\boldsymbol{U}_{\infty}$

$U_{V}$

$U_{W}$

$U_{G S}$

W

$w M$

woWS

wTS

$w W$

$W B$

WR

$y^{+}$

$\varepsilon$

$\rho$

$\rho_{\infty}$

$\mu$

$\mu_{t}$

$\omega_{W}$
Static pressure

Total pressure

Reference pressure

Smooth underbody

Temperature

Flow velocity

Flow velocity component in $\mathrm{y}$-direction

Flow velocity component in z-direction

Freestream velocity, relative air speed

Vehicle velocity

Wind velocity

Freestream velocity

Reference car width, wheeltrack

with mirrors

With no wheel struts

With top strut

with wheels and wheelhouses

Wheelbase distance

Wheel radius

Non-dimensional distance from the wall up to closest cell

Turbulence dissipation rate

Air density

Reference air density

Dynamic viscosity

Turbulence viscosity

Rotation rate of the wheels 


\section{Appendix}

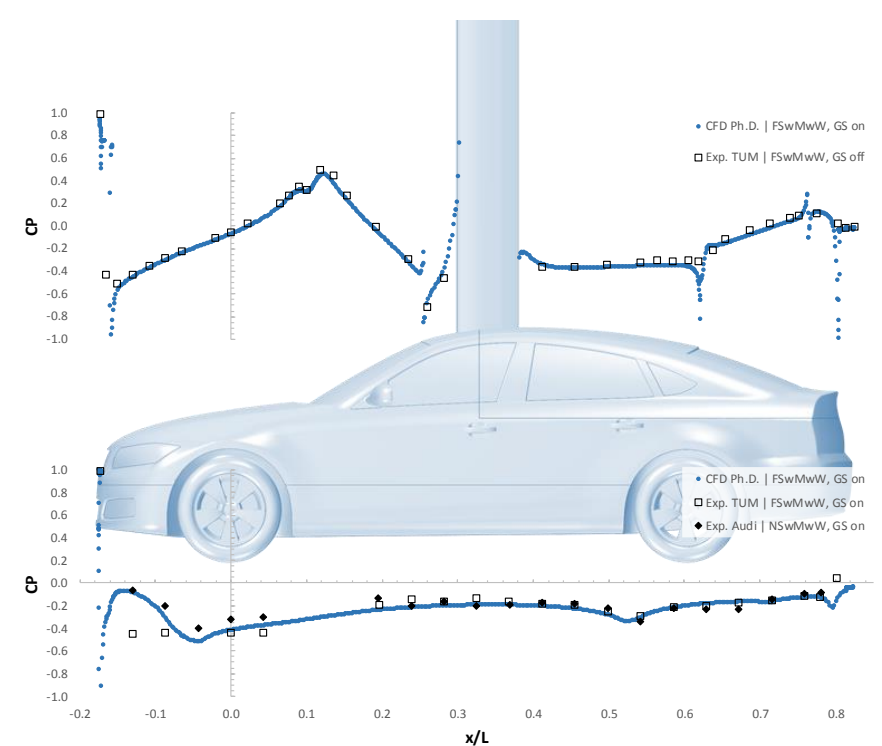

(a)

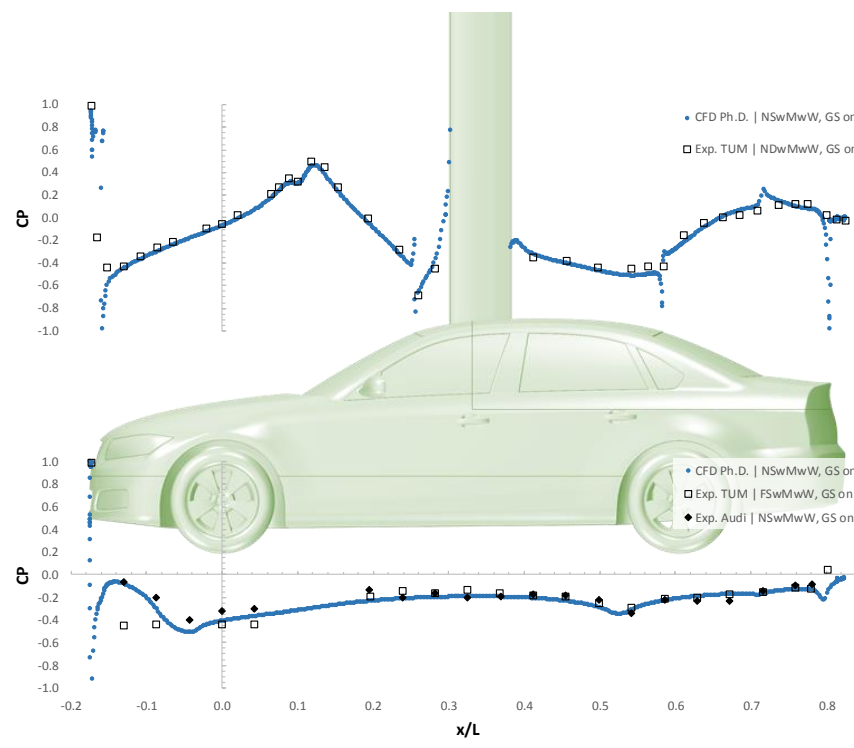

(b)

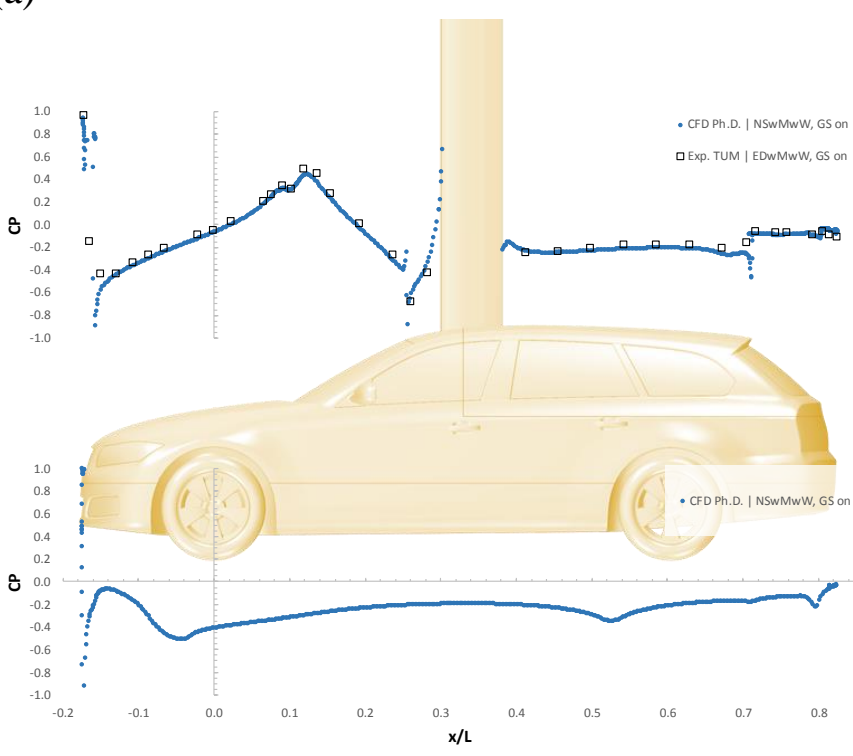

(c)
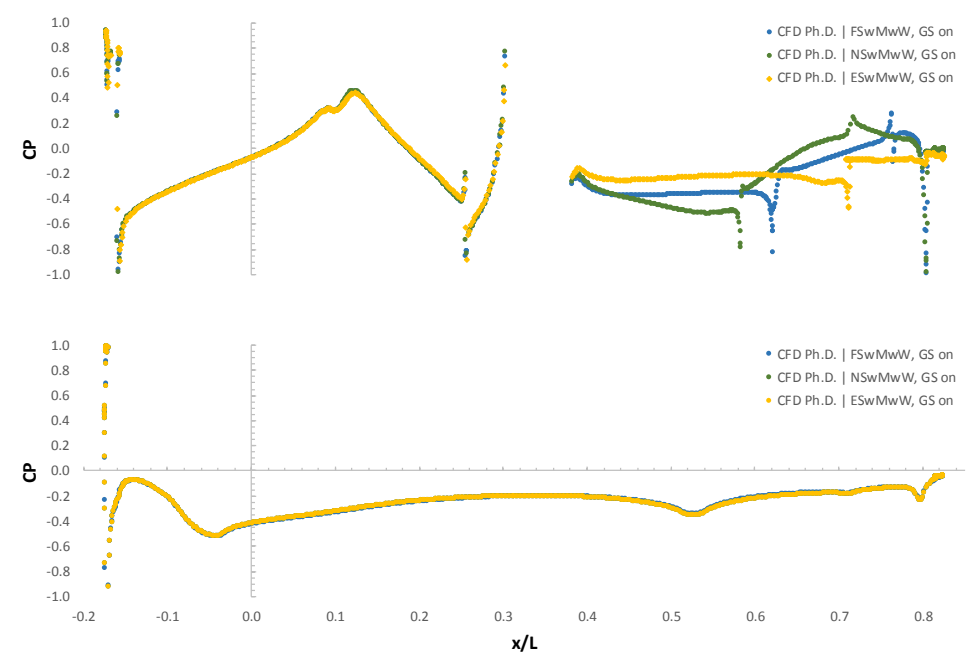

(d)

Figure 8. Pressure coefficient (upper and lower surfaces) from the validation process: (a) Fastback, (b) Notchback, (c) Estateback, and (d) superposition of the three car variants. The models are in ground simulation cases, and include smooth underbody, side mirrors and wheels, and top strut (_SwMwW, GS on, wTS).

Page 11 of 13 


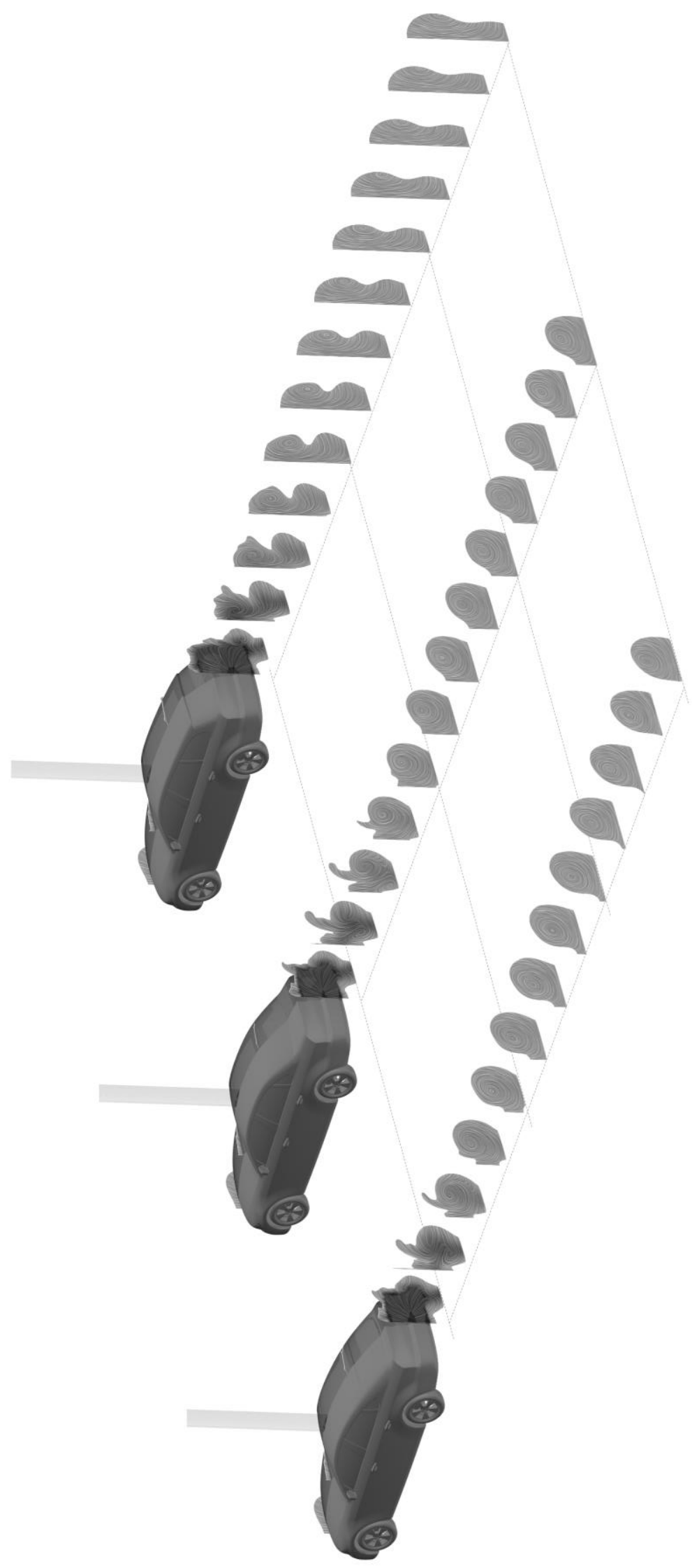

Figure 9. Wake defined by velocity magnitude $(u)$ : Fastback (at left), Notchback (at the middle), Estateback (at right) car variants. The models are in ground simulation cases, and include smooth underbody, side mirrors and wheels, in an open road environment (_SwMwW, GS on, wTS). The velocity fields are clipped with maximum threshold of $0.9 U_{\infty}$. 


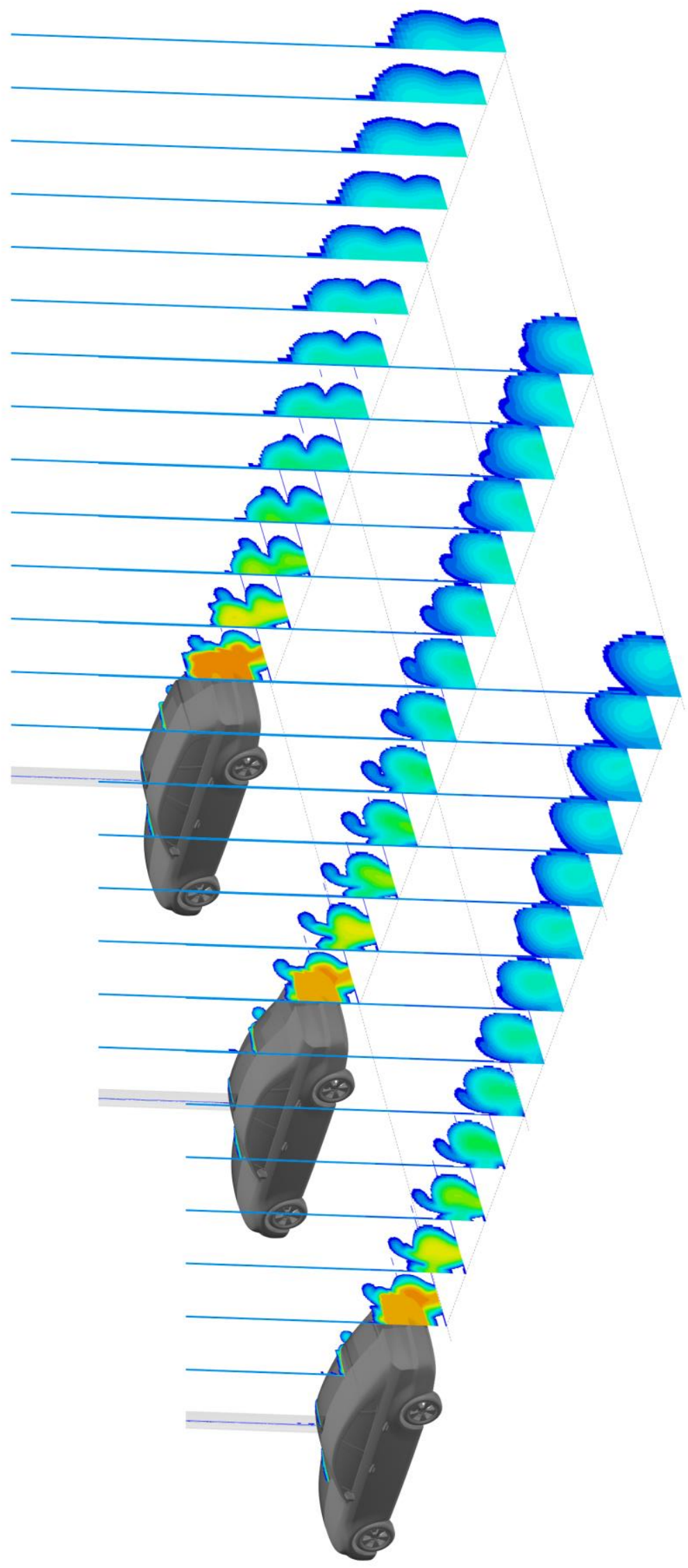

Figure 10. Wake defined by total pressure loss coefficient $\left(C_{P_{T L}}\right)$ : Fastback (at left), Notchback (at the middle), Estateback (at right) car variants. The models are in ground simulation cases, and include smooth underbody, side mirrors and wheels, in an open road environment (_SwMwW, GS on, wTS).

Page 13 of 13 
2017-03-28

\section{Comparison of the far-field aerodynamic wake development for three DrivAer model configurations using a cost-effective RANS simulation}

Soares, Renan F.

Society of Automotive Engineers

Renan F. Soares, Kevin P Garry and Jennifer Holt. Comparison of the far-field aerodynamic wake development for three DrivAer model configurations using a cost-effective RANS simulation. WCX17: SAE World Congress Experience, 4 - 6 April 2017, Detroit, Michigan, USA. http://papers.sae.org/2017-01-1514/ Downloaded from Cranfield Library Services E-Repository 